\title{
Preface
}

\section{Remote Monitoring and Physiologic Sensing Technologies}

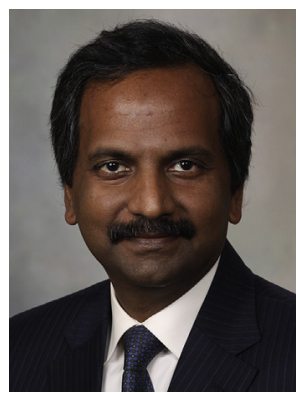

Samuel J. Asirvatham, MD

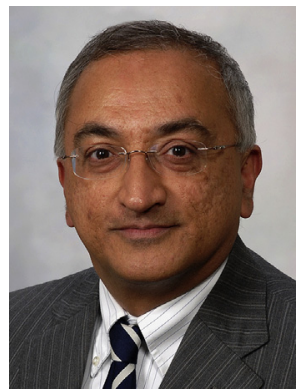

K.L. Venkatachalam, MD Editors
Modern methods of cardiovascular monitoring encompass a vast array of sensing, transmitting, and processing technologies that have variable utility depending on the physiologic parameter and disease process being evaluated. As the population continues to age and technology improves-both by miniaturization of existing hardware and by improvement in the ability to collect, process, and transmit real-time information for a variety of physiologic variables - the options to treat patients in the ambulatory setting will continue to evolve. The cardiac electrophysiologist will often be responsible for the choice of monitoring technology, the implantation or implementation of it, and the interpretation of the resultant data. Furthermore, with improved availability of these technologies, both physicians and patients will continue to encounter an increasing ability and desire to treat or monitor patients at home, which will require a thorough understanding of current options and their corresponding indications

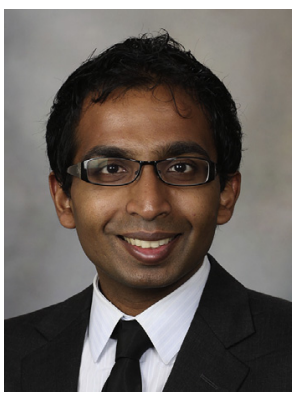

Suraj Kapa, MD

and limitations. Thus, a thorough understanding of remote monitoring technologies and the data underlying their use is critical to the modern electrophysiologist.

In this issue of Cardiac Electrophysiology Clinics, we focus on remote monitoring, including the history underlying its development, the current state of the technology, and potential future routes of development and study. Also found in this issue are current data on the utility of existing remote monitoring technologies in disease processes ranging from atrial fibrillation to stroke. Also presented are state-of-the-art, not yet clinically available technologies to widen the scope of the reader on what the future of these technologies may hold. Thus, through this edition of the Cardiac Electrophysiology Clinics, the reader may expect to attain a comprehensive outlook on the current state of remote monitoring and physiologic sensing technologies along with perspectives on how they 
may be utilized in current and future clinical practice and research.

Samuel J. Asirvatham, MD Department of Internal Medicine and Pediatric Cardiology St Mary's Hospital Mayo Clinic College of Medicine 200 First Street SW Rochester, MN 55905, USA

K.L. Venkatachalam, MD Department of Internal Medicine Division of Cardiology
Mayo Clinic College of Medicine 4500 San Pablo Road, Davis 7 Jacksonville, FL 32224, USA

Suraj Kapa, MD Division of Cardiology Mayo Clinic College of Medicine 200 First Street SW Rochester, MN 55905, USA

E-mail addresses: asirvatham.samuel@mayo.edu (S.J. Asirvatham) venkat.KI@mayo.edu (K.L. Venkatachalam) Kapa.Suraj@mayo.edu (S. Kapa) 